\title{
EL PROCESAMIENTO DE LAS RELACIONES LÉXICAS
}

\author{
CARMen VARO VARO \\ Universidad de Cádiz
}

\begin{abstract}
RESUMEN
En este trabajo analizamos las relaciones léxicas tomando como punto de partida el principio de contraste, por constituir este el eje central sobre el que estas relaciones se articulan tanto en los desarrollos de Lingüística general como en los de Psicolingüística. Con objeto de profundizar en el procesamiento de la antonimia, la parasinonimia y la hiperonimia-hiponimia, tras llevar a cabo un examen de las ventajas e inconvenientes de los enfoques léxico y conceptual, proponemos aquí una caracterización psicolingüística de estas, basada, especialmente, en la indagación de los dominios cognitivos implicados en ellas y en la actuación de una serie de mecanismos conceptuales, entre los que destaca la habilidad de focalización. Las diferencias observadas respecto al funcionamiento de estas relaciones en los ámbitos de la lengua y del hablar se ven reforzadas por los datos procedentes de la investigación de los procesos de adquisición, comprensión y producción léxicas.
\end{abstract}

Palabras clave: Relaciones léxicas, antonimia, sinonimia, hiperonimia-hiponimia, procesamiento léxico.

\section{ABSTRACT}

In this paper we will analyze lexical relations taking the principle of contrast as a starting point, since this is the axis which these relations are articulated on, not only in General Linguistics but in Psycholinguistics too. After reviewing both the advantages and disadvantages of the lexical and conceptual approaches, we propose a psycholinguistic characterization in order to study antonymy, parasynonymy and hyperonymy-hyponimy processing in depth. Such characterization is based on research on cognitive domains and on the performance of several conceptual mechanisms, especially of focalization. The differences noticed in the language system and in the speech are supported by data from research on the processes of lexical acquisition, comprehension and production.

Key Words: Lexical relations, antonymy, synonymy, hyperonymy-hyponymy, lexical processing. 


\section{EL PRINCIPIO DE CONTRASTE Y LAS RELACIONES LÉXICAS ${ }^{1}$}

\subsection{El principio de contraste en el marco de la Lingüistica estructural}

El principio de contraste es el eje central del concepto de «oposición», sin duda uno de los más interesantes y productivos, por sus aplicaciones en el análisis léxico de las lenguas, generado en el marco del estructuralismo lingüístico. En concreto, será fundamental la interpretación de este en el estructuralismo funcionalista de corte praguense, donde se entiende como principio general que permite la definición de cada unidad funcional del sistema y que implica la posibilidad de analizarlas en rasgos distintivos ${ }^{2}$.

Tal interpretación ocupa un lugar central en las investigaciones lingüísticas ${ }^{3}$, al tratarse de un concepto introducido en los albores del estructuralismo por F. de Saussure ${ }^{4}$. Para este autor, cada elemento será definido negativamente o por su «oposición recíproca en el seno de un sistema definido» (1980, p. 168). La relevancia de la relación de contraste es tan extrema que el concepto será reformulado posteriormente por Karčevskij 1929, pp. 88-93, autor que, frente a la negatividad manifiesta en la caracterización saussureana, otorga especial importancia a la existencia de una base común.

El análisis a partir de marcas opositivas fue utilizado por primera vez, como aplicación práctica en el ámbito de la Fonología, por los componentes del Círculo Lingüístico de Praga. La marcabilidad, frecuentemente interpretada como relación asimétrica entre los dos polos de una oposición, es un concepto clave en los desarrollos fonológicos de N. Trubetzkoy y R. Jakobson en los años 30. El primero de ellos elaboró en sus Principios de Fonología una clasificación lógica de las oposiciones fonológicas distintivas, atendiendo a diversos criterios, entre los que destaca el basado en las relaciones de contraste entre los términos de la oposición, que sirve para distinguir entre «privativas», «graduales» y «equipolentes». Trubetzkoy 1973, p. 60 reconoce, además, la validez de su extensión a otros niveles lingüísticos señalando que:

\footnotetext{
1 Entenderemos aquí «relación léxica» como relación establecida entre signos, tomando como punto de partida los significados de estos. Cf. Casas Gómez 1999 y 2005. En este punto nos alejamos de otros autores que aluden con esta designación exclusivamente a las relaciones entre significantes de signos.

${ }^{2}$ Existe una segunda interpretación del concepto de «oposición», más específica, que se circunscribe a determinados estudios lingüísticos que emplean el término en el ámbito de las relaciones léxicas. Cf. Varo Varo 2006, pp. 823-826.

${ }^{3}$ El devenir de esta interpretación de «oposición» en los estudios de lingüística, desde Trubetzkoy hasta el estructuralismo americano, es sintetizado por Marcus 1989, pp. 943-961.

${ }^{4}$ El lingüista ginebrino es el primero en proponer un estudio sistemático de las oposiciones de las lenguas, vinculado a la naturaleza discreta de sus unidades (1980, p. 168).
} 
Deben establecerse, ante todo, ciertos conceptos que son de importancia decisiva no solo para los sistemas de oposiciones fonológicas sino en general para todo tipo de oposiciones.

Frente a la anterior clasificación tripartita, R. Jakobson, en una comunicación pronunciada en 1939 en el Tercer Congreso Internacional de las Ciencias Fonéticas (Gante), presenta por primera vez su teoría binarista $^{5}$ (cf. Jakobson 1975), en la que las relaciones fonológicas de las lenguas se explican por la presencia o ausencia de un determinado rasgo distintivo, que puede manifestarse como elección entre dos cualidades polares de una misma categoría o bien la presencia / ausencia de una misma cualidad.

El análisis en rasgos de contraste, inspirado en las teorías fonológicas praguenses ${ }^{6}$, fue progresivamente adaptado a los significados léxicos a través del análisis componencial. Aplicado a la indagación de los campos léxicos, fue adoptado por lingüistas de diferentes posturas teóricas. No obstante, los semantistas elaboran sus análisis del significado basándose en «semas» o rasgos de contenido mínimo, portadores de sustancia sémica. Estos resultan más complejos que los rasgos distintivos fonológicos, pues en ellos se combinan aspectos funcionales (hechos de lengua) y hechos de la experiencia real de los hablantes (rasgos enciclopédicos). Así pues, el objeto de investigación de la semántica y el léxico se presenta mucho más complicado que el objeto de la fonología. Por ello, como advierten algunos (entre ellos, Wotjak 1979, pp. 108 y ss., y Waugh 1982, p. 299), los métodos fonológicos han de utilizarse con suma precaución en la investigación de la estructura semántica.

En este marco de análisis, sobre todo, las denominadas oposiciones «privativas ${ }^{7}$ » ofrecen problemas en cuanto a su caracterización desde pa-

${ }^{5}$ En esta propuesta el concepto de oposición adquiere un carácter lógico y es trasladado desde el fonema al rasgo distintivo, con lo que se resuelve el problema de la definición negativa mantenida por Saussure. De este modo el valor negativo no está en el fonema sino en sus componentes.

${ }^{6}$ Este tipo de análisis fue introducido por primera vez en el ámbito de la semántica por L. Hjelmslev en 1943. En concreto, será en sus Prolegómenos a una teoría del lenguaje donde defiende, por primera vez, la posibilidad de descomponer el significado en elementos de contenido menores (cf. ed. esp. 1971, p. 102). Su comparación entre semántica y fonología se funda en la suposición de que ambas ocupan un lugar limítrofe en la lingüística respecto a otras disciplinas extralingüísticas. Cf. Wotjak 1979, p. 107.

${ }^{7}$ La clasificación de las oposiciones léxicas y la caracterización de cada uno de los tipos propuestos de acuerdo con el modelo de la Fonología encuentra también problemas en las oposiciones graduales, que en la clasificación de Trubetzkoy 1973, p. 67 «son aquellas cuyos miembros se caracterizan por que presentan en distinto grado una misma particularidad», pues entran en contradicción, en cualquiera de los niveles lingüísticos, con uno de los principios fundamentales del estructuralismo funcionalista: el de discreción. Efectivamente, si bajo el prisma de la realidad extralingüística es fácil establecer grados, otra cosa son las relaciones estrictamente lingüísticas entre unidades discretas. 
rámetros semánticos, al existir en semántica dos interpretaciones bastante dispares de la noción de «privatividad»: una de ellas, de carácter funcional, basada en la indiferencia de uno de los miembros de la oposición respecto a una determinada marca, y otra, de naturaleza sustancial, basada en la presencia en uno de los miembros de la oposición de una marca ausente en el otro miembro. Mientras que la primera de estas concepciones se ajusta a criterios estrictamente lingüísticos, la segunda resulta inadecuada por extralimitar el marco de los hechos semánticos.

En el ámbito de las relaciones léxicas, como producto del apego al binarismo, la relación antonímica es explicada con frecuencia a partir de una oposición cualitativa, organizada mediante dos polos opuestos bajo un eje «semántico» único, de naturaleza sustancial. Desde esa óptica, la presencia o ausencia es interpretada en términos de la presencia (en alto, grande, etc.) o ausencia (en bajo, pequeño, etc.) de un determinado valor positivo. Resultado de este análisis cualitativo será, además de una interpretación errónea del concepto de privatividad, la conversión de un gran número de oposiciones equipolentes, las antonímicas, en privativas. Por otra parte, como resultado final del objetivo de reducir a oposiciones binarias todas las relaciones léxicas, quedan sin explicación ciertas relaciones sinonímicas ${ }^{8}$, en concreto la cuasisinonimia o parasinonimia (tomo /volumen, valiente / temario, etc.), que solo encuentran, al igual que la antonimia, su justificación en la equipolencia. Como consecuencia, sería más bien oportuno distinguir una tipología general de oposiciones equipolentes según esta afecte a antónimos o parasinónimos y con posibles modalidades en cada uno de estas relaciones, frente a la oposición de privatividad, vinculada a la relación de hiperonimia-hiponimia ${ }^{9}$.

\subsection{El principio de contraste en el marco de la Psicolingüística}

En el marco de la Psicolingüística evolutiva el principio de contraste constituye una de las bases pragmáticas explicativas de la adquisición

\footnotetext{
${ }^{8}$ No incluimos la sinonimia absoluta en el conjunto de las relaciones léxicas, por constituir esta un hecho de variación y no afectar, por tanto, a dos unidades léxicas distintas, que comparten una parcela de su significación, sino a un único contenido semántico que puede ser representado materialmente a través de dos expresiones.

${ }^{9}$ Esta relación ha sido particularmente identificada con la sinonimia en la semántica de Coseriu 1981, p. 224, que caracteriza esta última relación por estar sujeta a la privatividad funcional o verdadera privatividad. A este respecto, debemos aclarar que los por él llamados «sinónimos», ejemplificados con pares del tipo dominer / maîtriser, albus / candidus, dissiper / gaspiller, son en realidad fruto de la neutralización de una relación de hiperonimia-hiponimia, como advierte Casas Gómez 1997, pp. 99-106. En el primer ejemplo el término no marcado sería dominer, frente al marcado maîtriser, que posee un sema más: «voluntariamente», con lo que el no marcado puede aparecer cuando media una neutralización en lugar del marcado, convirtiéndose ambos en sinónimos contextuales.
} 
del léxico. En este sentido, Clark 1987 y 1995 lo convierte en mecanismo esencial para justificar la diferenciación semántica efectuada por el niño cada vez que se enfrenta a un nuevo significante aplicado en un mismo contexto:

Contrast, then, captures the insight that when speakers choose an expression, they do so because they mean something that they would not mean by choosing some alternative expression. Speaker choices in any domain mean what they do in part because they contrast with their options both in that domain and in the language as a whole. The major result of this is that speakers do not tolerate complete synonyms (1995, p. 70).

Este enfoque, compartido por otros autores como Bolinger 1977, p. 1, partidario de que «if two ways of saying something differ in their words or their arrangement, they will also differ in meaning», se remonta a la postura defendida por M. Bréal en sus trabajos ${ }^{10}$ sobre las leyes intelectuales del lenguaje, en los que se establecen algunas de las bases fundamentales de la Semántica como ciencia de la significación y de sus cambios. En concreto, la denominada ley de repartición de sinónimos mostraba la inestabilidad de dicha relación y la tendencia por parte de la comunidad de hablantes a especializar los significados de las unidades léxicas inicialmente sinónimas. Desde estos presupuestos la relación de sinonimia se torna, por tanto, un fenómeno inestable en las lenguas, por entrar en contradicción con la tendencia a la economía lingüística y cognitiva, inclinada al abandono del estado de la identidad perfecta de las palabras. Desde planteamientos más cercanos a la Pragmática, Grice 1975 justifica este fenómeno a través del principio de cooperación. Desde este, se considera que el uso en el discurso de una unidad sinónima puede aportar información relevante o distinta.

Asimismo, el principio de contraste ha sido tomado en consideración en diversos estudios de carácter experimental interesados en extraer diferencias en el orden de adquisición de las relaciones léxicas ${ }^{11}$, en los que se pone de manifiesto que el niño adquiere las estructuras y funciones lingüísticas más simples (como, por ejemplo, el par grande / pequeño y, de las dos unidades léxicas, antes grande), para posteriormente llegar a las más complejas (pues, alto / bajo y ancho / estrecho, suponen una dimensión más específica, sobre todo el segundo miembro). En dicho proceso se observa no solo que es la complejidad cognitiva el factor determinante del orden de adquisición ${ }^{12}$, como queda patente en los

${ }^{10}$ Cf., en este sentido, Bréal 1897, p. 30.

${ }^{11}$ Cf., por ejemplo, Feider 1973, Landis, Herrman y Chaffin 1987, Aprile 1992 y Hatzivassiloglou 1999.

${ }^{12}$ Krampen 1984, pp. 173-174, aplicando esta idea a los conceptos espaciales mediante dibujos infantiles, comprobó la existencia de rasgos binarios (o graduales) operativos que dife- 
usos y las sustituciones realizados por el niño (cf. Clark 1972, p. 751), sino también que las relaciones de contraste aparecen a muy temprana edad, en tanto que el niño percibe las semejanzas más tardíamente (cf. Varo Varo 2003, pp. 16-18).

En el ámbito de los estudios sobre el procesamiento léxico, encontramos posturas como la mantenida por Murphy 2003, p. 26, autora que descarta la relevancia de las relaciones sinonímicas y antonímicas para la competencia léxica y las hace derivar del principio de relación "por contraste», a partir del conocimiento conceptual o enciclopédico de los hablantes. De acuerdo con este principio, entendido en este caso en un sentido amplio que abarca sinónimos, antónimos, hiperónimos, etc., dos elementos son relacionados, si mínimamente son diferenciados de una forma contextualmente adecuada:

The contrast relation holds among the members of a set if: they have all the same contextually relevant properties but one (Murphy 2003, p. 44).

La relevancia del principio de contraste ${ }^{13}$, frente a la búsqueda de similitud, ya fue constatada por Chaffin y Herrmann 1984, pp. 134-141 mediante distintas tareas relacionales en las que esperaban que los sujetos agruparían entidades similares u objetos con atributos similares (rake-fork, movie-photograph) como sinónimos y estos, por el contrario, los asociaron como antónimos.

Finalmente, atendiendo a su correlato biológico, a partir de ciertas investigaciones basadas en la neuroimagen mediante técnicas como PET y fMRI, se han formulado teorías interpretativas, señaladas por Ullmann 2006, p. 259, como la hipótesis semántica, desde la que se postula que el cerebro está organizado anatómicamente de acuerdo con diferencias semántico-conceptuales ${ }^{14}$. En ese sentido, el principio de contraste serviría de eje vertebrador no solo de diversas categorías lingüísticas, sino también de distintas redes de relaciones semánticas ${ }^{15}$.

rencian significados transmitidos no verbalmente por el canal visual. Se constata, por tanto, un sistema de rasgos distintivos que se transforma a medida que progresa la habilidad para coordinar las operaciones mentales relacionadas con espacios de distintas dimensiones.

${ }^{13}$ Este principio entra en cierta contradicción con la propuesta prototípica, de gran influencia en el marco de la lingüística cognitiva, en la que solo se contemplan grados y no delimitaciones de carácter discreto que permiten contrastar significaciones.

14 Diversos estudios, como los realizados por Butters, Barton y Brody 1970, Caramazza y otros 1976, han mostrado la relación de determinadas capacidades para el establecimiento de oposiciones por contraste con ciertas áreas cerebrales, como el giro angular.

${ }^{15}$ En estos estudios se ha mostrado también la estrecha vinculación entre funciones lingüísticas y otras funciones cognitivas por su dependencia común de determinadas estructuras cerebrales. Cf. Damasio y otros 1996. 


\section{LOS ENFOQUES LÉXICO Y CONCEPTUAL EN LOS ESTUDIOS SOBRE EL PROCESAMIENTO DE LAS RELACIONES LÉXICAS}

En la investigación psicolingüística sobre el léxico, uno de los temas que más controversia suscita es la representación y configuración del lexicón ${ }^{16}$. En relación con ello interesa determinar cuáles son las entradas léxicas, su estructura interna y su organización dentro del lexicón. En este último punto, relativo a la configuración de los conocimientos léxicos, existen opiniones muy diversas sobre si las relaciones léxicas están mentalmente fijadas o no. Si descartamos, como respuesta probable, que estemos programados de forma innata con el conocimiento de todas ellas, dado que cada lengua es la responsable de este, podemos pensar que esta información lingüística es almacenada en la memoria a largo plazo, junto con otros conocimientos lingüísticos, o bien, que dicho conocimiento se genera cada vez que es necesario ${ }^{17}$, a partir de un conjunto de reglas y principios de carácter más general. En suma, la falta de unanimidad en cuanto a la propia naturaleza de las relaciones léxicas y, como consecuencia, de su estatus en el lexicón se percibe en la extrema división de posturas teóricas al respecto.

\subsection{El enfoque léxico de la antonimia}

Entre estas posturas, queremos destacar, en primer lugar, la mantenida por aquellos que enfocan la relación antonímica desde una perspectiva léxica, reservando el enfoque conceptual para la sinonimia y la hiperonimia-hiponimia. Desde este prisma se establecen, por tanto, claros límites entre información léxica y conceptual, y se otorga a las relaciones antonímicas una naturaleza distinta frente al resto de relaciones, por considerar que en las primeras la información lingüística se genera contextualmente, tal vez facilitada por patrones de frecuencia (repetición, coocurrencia de estructuras o combinaciones, etcétera).

${ }^{16} \mathrm{Al}$ margen de este tema, destacan otras dos grandes preocupaciones entre los investigadores del procesamiento léxico. La primera, vinculada al problema del reconocimiento, es describir cómo se localiza la entrada apropiada y cómo se llega a la información léxica a partir de la información sensorial y contextual. La segunda es analizar cómo se combinan e integran los diferentes tipos de información léxica almacenados para lograr construir una representación significativa.

17 Como opción más plausible, Murphy 2003, pp. 6-7 señala que probablemente hemos aprendido que las palabras se relacionan sinonímica o antonímicamente y hemos añadido dicho conocimiento a nuestras representaciones mentales: «So while all semantic relations among words can be generated via a single relational principles, this does not mean that the relations have to be generated each time they are handed». 
Esta línea es desarrollada por G. Miller y otros colaboradores desde hace años en el modelo conocido como WordNet ${ }^{18}$, entendido como sistema de referencia léxica basado en principios psicolingüísticos. Se trata de un diccionario on line diseñado para reflejar la organización de la memoria léxica y resolver tareas reales relacionadas con la categoría adjetivo. Este particular enfoque tiene como punto de partida la observación del comportamiento de las categorías verbales respecto a las relaciones léxicas. Así, se aprecia que generalmente los sustantivos carecen de antónimo canónico y su organización semántica se apoya más en la relación de hiperonimia-hiponimia. Frente a estos, los adjetivos se organizan frecuentemente a través de la relación de antonimia. Incluso, como advierten Gross, Fischer y Miller 1989, pp. 92-106, cuando para determinados adjetivos no existen antónimos canónicos, a cualquier hablante le resulta más fácil proporcionar un antónimo no canónico para estos que para un sustantivo. Por otra parte, a pesar de que en esta base de datos las unidades léxicas de significado similar se organizan en conjuntos de sinónimos, dicha relación no se toma como base organizadora del léxico, por tener algunos sinónimos distintos antónimos (por ejemplo, los sinónimos rise y ascend del inglés se relacionan antonímicamente con fall y descend, respectivamente). Precisamente, tales casos de sinonimia «intransitiva», siguiendo la denominación de Miller y otros 1990, p. 242, sirven de base a autores como Gross, Fischer y Miller 1989 para defender la antonimia como relación entre lexemas y no entre conceptos, de modo que la relación conceptual solo se puede entender como reflejo semántico, es decir, siempre como efecto y nunca como causa de la relación entre formas léxicas.

Por otra parte, para los partidarios de este enfoque una definición puramente semántica de una relación como la antonimia no explica la tendencia a establecer con mayor frecuencia esta relación entre determinados significantes que los hablantes identifican de forma común (por ejemplo, bueno / malo y no bueno / malévolo). En esta línea se ubica la postura teórica de Justeson y Katz 1991, pp. 138 y 142, pioneros en este tipo de acercamiento, que resuelven el supuesto problema definiendo la antonimia como relación específica entre palabras y no entre conceptos (1991, p. 138). En su opinión, «[antonyms] occur in parallel and often essentially identical phrases».

Para corroborar este enfoque surgen numerosos trabajos de tipo práctico destinados al estudio de los pares antonímicos atendiendo a su coocurrencia en los textos, como los llevados a cabo por Mettinger ${ }^{19}$

18 Una discusión sobre los problemas de la noción de antonimia presente en WordNet y defendida por Miller y otros colaboradores puede verse en Murphy y Andrew 1993, pp. 304 y ss.

19 No obstante, Mettinger 1994, p. 169, al analizar los denominados «opuestos en contexto", a partir de un amplio corpus de novelas inglesas, descubre que no siempre los hablantes conciben como antónimos determinados pares que contrastan contextualmente con cierta asiduidad (cat/mouse, murder/suicide). 
1994, Fellbaum 1995, Kwon 1998 o, más recientemente, Jones ${ }^{20}$ 2002, entre otros. Por su parte, Fellbaum 1995, p. 286, siguiendo dicha propuesta, considera que los antónimos son aprendidos por su frecuente coocurrencia en las lenguas dentro de determinados marcos sintácticos sin posibilidad de ser sustituidos unos por otros. En semejantes términos Muehleisen 1997 encuentra en ciertos perfiles combinatorios repetidos la clave para la explicación de la antonimia entre pares como dry / wet. Otro de los argumentos aducidos en este sentido por Miller y Fellbaum 1991, p. 211 reside en el carácter primario de la relación morfológica presente en los antónimos gramaticales, frente a la relación semántica, que solo se explica, desde estos planteamientos, como consecuencia de la anterior.

Las respuestas para justificar la naturaleza léxica de la antonimia, sustentadas básicamente, como hemos visto, en la frecuente coocurrencia de antónimos en el hablar han sido rebatidas, por resultar insuficientes, al coocurrir también con asiduidad otras unidades no relacionadas antonímicamente. En esta línea, se ha señalado que más bien es la relación conceptual la que trae como consecuencia la vinculación léxica y no a la inversa, y que, en cualquier caso, limitar la relación antonímica a una mera explicación léxica dificulta la descripción de sus propiedades semánticas (cf. Murphy y Andrew 1993, p. 304).

Otro problema que plantea el enfoque léxico de la antonimia, además de no tenerse en cuenta la polisemia de las unidades implicadas, es que en él se ignoran determinadas asimetrías desde el punto de vista de los significados, que, como observamos en Varo Varo 2007, pp. 182-207, llevan a elegir unas unidades frente a otras. Por consiguiente, pensamos que no es la asociación entre significantes lo que justifica la relación semántica, sino, al contrario, es justamente la cercanía semántica desde los puntos de vista paradigmático y sintagmático la que conduce a la vinculación entre los significantes de tales signos.

Por otra parte, al margen de las dificultades que entraña la adopción del significante ${ }^{21}$ como punto de partida de este enfoque, hemos de subrayar también la falta de delimitación entre contexto de habla y contexto de lengua. En este sentido, consideramos que la dimensión sintagmática de los signos forma parte de su significado $y$, por tanto, puede actuar como criterio para los hablantes en la elección de las unidades léxicas al construir un enunciado. Por último, autores como Miller y otros 1990, p. 240 y Miller y Charles 1991 adoptan una interpretación muy laxa de sinonimia, más próxima a la lógica que a la Lin-

${ }^{20}$ Jones 2002, p. 23 considera insuficiente la definición semántica de la relación: «Any definition of antonymy should be lexical as well as semantic».

${ }_{21}$ Para un estudio de la problemática del concepto de significante presente en el funcionalismo semántico, cf. Casas Gómez 2008. 
güística, desde la cual dos unidades son sinónimas si pueden alternar en un contexto sin cambiar los valores de verdad. En tal interpretación no se distinguen significado, sentido y referente.

\subsection{El enfoque conceptual de las relaciones semánticas}

Frente a esta perspectiva, en la que la antonimia se separa del resto de relaciones, otros investigadores, como Murphy y Andrew 1993, defienden la naturaleza conceptual de todas las relaciones semánticas. Desde sus planteamientos, aunque la coocurrencia refuerza los vínculos establecidos en la memoria semántica, la base conceptual es primaria.

Tal interpretación se ubica en el marco de la denominada semántica conceptual, una de las principales orientaciones de la semántica cognitiva $^{22}$, junto con la semántica prototípica, que, siguiendo los planteamientos de Jackendoff 1983, establece una equivalencia exacta entre estructura conceptual y estructura semántica, de forma que el análisis semántico debe partir del análisis de las representaciones mentales. En este sentido, se parte de la existencia previa de conceptos primitivos que posteriormente son aplicados para la interpretación y categorización de diversas experiencias, a través de una serie de principios vinculados a la combinación conceptual. Los significados léxicos, por tanto, se construirían a partir de nuestra experiencia del mundo y de la lengua. Esta particular concepción de los significados ha sido bastante criticada por no aportar suficientes evidencias que apoyen el paralelismo perfecto entre significados y conceptos (cf. Singleton 2000, p. 79).

Los partidarios de la aplicación de este enfoque para la caracterización de las relaciones léxicas se apoyan en el fenómeno que designan como «lexical preemption». Así, para Murphy y Andrew 1993, p. 305 la razón por la que small no es un buen antónimo de big es que ya existe la palabra little (cf. también Lehrer 1990). Añaden, asimismo, razones de tipo estilístico (little es informal, frente a small que es formal). Paralelamente, se apoyan en el «modelo de inestabilidad de las relaciones conceptuales» de Barsalou 1987, según el cual los juicios sobre las relaciones conceptuales básicas pueden variar entre sujetos y entre contextos. Desde esta perspectiva, si diferentes contextos inducen a producir diferentes respuestas ante los mismos estímulos, en el caso, por ejemplo, de los adjetivos, los diferentes sustantivos con que se combinan pueden modificar o seleccionar partes diferentes de la representación del adjetivo en cuestión que determinen la vinculación con distintos antónimos o sinónimos.

${ }^{22}$ Tanto la semántica conceptual como la prototípica constituyen alternativas surgidas en el marco de la investigación cognitiva del significado, que tratan de superar algunos de los problemas planteados, especialmente, en la semántica componencial. 
Partiendo de tales consideraciones, a través de varias pruebas experimentales, destinadas al examen de la producción y la comprensión de sinónimos y antónimos canónicos y no canónicos en distintos tipos de contextos, tras constatar que la interpretación de las unidades léxicas es diferente según se inserten en unas estructuras u otras o aparezcan de manera aislada, Murphy y Andrew 1993 pp. 315 y ss., concluyen que tanto la sinonimia como la antonimia son relaciones conceptuales, porque cuando cambian los contextos, también cambian los sinónimos y antónimos proporcionados por los individuos que participaron en las pruebas. Mantienen, en esta línea, que estas unidades no necesitan ser prealmacenadas, pues pueden ser procesadas a través de la similitud semántica:

....antonyms do not need to be prestored - they can be generated by choosing other words with similar meanings and searching for one that differs only in one dimension (1993, p. 317).

A esta propuesta conceptual hemos de objetarle, igual que en el caso de la propuesta léxica, la exclusión de la dimensión sintagmática de las unidades como parte integrante de su significado, que no debe confundirse con su combinatoria de habla, además de la falta de explicación en relación con los mecanismos específicos a través de los cuales accedemos conceptualmente a sinónimos y antónimos (pues ya se descarta el camino léxico). Por otra parte, en ella el principio de similitud se convierte en el eje esencial de las relaciones semánticas, cuando, como hemos visto más arriba, el principio de contraste es mucho más eficaz cognitivamente.

Pero, fundamentalmente, hemos de resaltar, a pesar de la importancia reconocida de la base cognitiva de las relaciones léxicas, la necesidad de establecer límites precisos entre los ámbitos conceptual y lingüístico. Así, aunque estas relaciones se pueden analizar como manifestación léxica de determinadas estructuras conceptuales, afectan a unidades léxicas que son intralingüísticas, es decir, dependen del sistema lingüístico en el que se inserten. En este sentido, hay que deslindar de las relaciones conceptuales, sin renunciar a su base cognitiva, las relaciones establecidas, en el seno de los sistemas léxicos particulares, entre significados de signos que comparten una base sémica común y presentan una serie de rasgos diferenciales, y cuya diversa articulación depende de la organización concreta de dichos sistemas.

Una vez situados en el ámbito de los hechos del lenguaje, siguiendo la propuesta de Casas Gómez 2002, es posible abordar las relaciones léxicas desde la perspectiva de la lingüística de la lengua o bien desde la de la lingüística del hablar, cada una de ellas con sus correspondientes implicaciones. En la primera, que nos remite al concepto de significado o contenido lingüístico formalizado intralingüísticamente 
en las lenguas particulares y base semántica del análisis estructural y funcional de estas lenguas, hemos de concentrar nuestro interés en aquellos pares de elementos léxicos entre cuyos significados podemos establecer una oposición en términos estrictamente sistemáticos, es decir, relación basada en la existencia de una base semántica común, junto a determinados rasgos o marcas diferenciales. En cambio, desde la perspectiva del hablar encontramos relaciones de similitud y contraste de sentidos, generadas a partir de determinadas inferencias condicionadas por el contexto en que aparecen insertos los elementos léxicos implicados.

\section{BASES PARA LA CARACTERIZACIÓN PSICOLINGÜÍSTICA DE LAS RELACIONES LÉXICAS}

Como hemos visto hasta ahora, en los estudios lexicológicos desarrollados tanto por la Lingüística estructural como por la Lingüística cognitiva, las relaciones léxicas son descritas y analizadas a partir de la convivencia en el marco de los significados léxicos de las propiedades semánticas de similitud y contraste. De hecho, en la denominada «semántica de rasgos» dichas propiedades constituyen la base para llegar a la definición de cada unidad del sistema de una lengua. De este modo, la sinonimia, la antonimia y la hiperonimia-hiponimia, por su importancia para la estructuración léxica de las lenguas, han sido caracterizadas, tomando como punto de partida el plano del contenido, como relaciones de significación o establecidas entre significados de signos ${ }^{23}$, a los que se somete a descomposición sémica para indagar las oposiciones en las que participan.

No obstante, resulta llamativa la falta de diferenciación clara de estas relaciones, sobre todo en el campo de la Lexicografía, donde sinonimia y antonimia constituyen relaciones opuestas, pues la primera consistiría en la mínima diferencia de significado y la segunda se referiría a la máxima diferencia de significación, sin apenas ahondar en otras posibles divergencias y semejanzas, ni en el grado de similitud o de contraste necesario para hablar de una u otra relación. La hiperonimiahiponimia o relación de inclusión semántica, como hemos tratado de mostrar, no siempre está bien diferenciada de la sinonimia ni de la inclusión real o referencial ${ }^{24}$.

\footnotetext{
${ }^{23}$ Frente a otras posturas teóricas, excluimos los hechos de polisemia y homonimia del marco de las relaciones léxicas. Para profundizar en el tratamiento de estos cf. Muñoz Núñez 1999.

${ }^{24}$ Por ejemplo, en casa / habitación existe inclusión real y en animal / perro la inclusión es semántica.
} 
La confusa interpretación de las relaciones léxicas conduce también a otros problemas, como la ausencia de clasificaciones suficientemente abarcadoras que aborden las diferentes manifestaciones de cada una de estas relaciones en los ámbitos de la lengua y del hablar. Por otra parte, en la mayoría de las caracterizaciones realizadas se observa el esfuerzo por aislar a los signos léxicos de los participantes en el evento comunicativo y de los procesos de producción y comprensión lingüísticas $^{25}$. En esta línea, consideramos que la Lingüística cognitiva, por su interés en los diversos mecanismos de conceptualización humana, basados fundamentalmente en la separación entre el individuo y el entorno que le rodea, el conocimiento en relación con la manipulación de objetos y la experiencia espacial y control de los movimientos, nos ofrece un marco explicativo de gran utilidad para descubrir los principales rasgos que singularizan a la antonimia, la sinonimia y la hiperonimiahiponimia léxicas.

En concreto, para analizar las diferencias desde el punto de vista psicolingüístico entre las relaciones léxicas, tomaremos como referencia, de un lado, la noción de «dominio cognitivo» ${ }^{26}$, entendido como representación mental de la forma en que se organiza el mundo, siguiendo la propuesta presente en Cuenca y Hilferty 1999, p. 70, y, de otro, el mecanicismo de la «focalización», en cuanto una de las principales habilidades presentes en los procesos de conceptualización.

El concepto de «dominio cognitivo», en estrecha conexión con la representación lingüística, presenta una estructura que ha sido descrita a menudo en términos de «esquema de imágenes» o «esquema topológico» ${ }^{27}$, en cuanto eje articulador de las propiedades que organizan nuestra experiencia y que coinciden en ciertas pautas de manifestación, vinculadas a nuestros movimientos en el espacio y la manipulación de objetos, de acuerdo con Johnson 1987. En él es común encontrar dos fuerzas dispuestas simétricamente que actúan respecto a un punto medio. En cualquier caso, se trata de un concepto manejado con asiduidad en la investigación psicolingüística, donde la mayoría de los autores concibe la estructura de un dominio en términos de un conjunto de dimensiones, vectores, categorías bipolares o algún tipo análogo de divi-

${ }_{25}$ Por ejemplo, la caracterización del par léxico comprar y vender será muy distinta, dependiendo de si se incluye o no el punto de vista de los protagonistas de las acciones implicadas. Si este no se tiene en cuenta, serán opuestos direccionales y si se incluye, serán inversos.

${ }_{26}$ Para la Lingüística cognitiva el individuo constituye un punto de referencia básico, de tal manera que un gran número de elementos de nuestro sistema conceptual están orientados con respecto a su semejanza o falta de semejanza con la persona prototípica, es decir, las distintas orientaciones conceptualizadas por el individuo partirán de sí mismo hacia fuera. Cf. Cooper y Ross 1975, pp. 174-175.

27 Se han utilizado otras denominaciones para este concepto, como «guiones» (scripts) y «marcos» $\mathrm{y}$ «configuraciones» (frames). Cf. Schank y Abelson 1977. 
sión, como señalan Gardner y otros 1978, p. 301. Respecto a sus aplicaciones en el ámbito de las relaciones léxicas, podemos señalar el modelo cognitivo de la antonimia diseñado por Cruse y Togia 1995, basado en el reconocimiento de dominios esquemáticos antonímicos de diversa índole, que originan sistemas monoescalares (long-short), biescalares equipolentes (cold-hot) o solapados (good-bad) e híbridos (clean-dirty).

Extendiendo este tipo de análisis a los tres tipos de relaciones léxicas, la antonimia podría explicarse como relación dentro de un dominio escindido en partes simétricas, la hiperonimia-hiponimia como relación de inclusión de dominios y la parasinonimia (sustentada en la equipolencia semántica) como relación entre dominios solapados. En este sentido, dos parejas léxicas como alto / bajo y hombre / mujer podrían analizarse como relación entre dos partes simétricas de los dominios dimensión y ser humano. En cambio, en los pares silla / mueble y oír / escuchar se analizaría el primer elemento como representante de un dominio incluido en el dominio representado en el segundo. Finalmente, los casos de pares como joven / nuevo y hospital / clínica ejemplifican dominios solapados o coincidentes en una parte de su esfera conceptual. Se explica, así, la paradójica proximidad entre antónimos, justificada por ocupar estos un mismo dominio, frente a la distancia entre cuasisinónimos, por ocupar éstos dominios, aunque próximos, distintos.

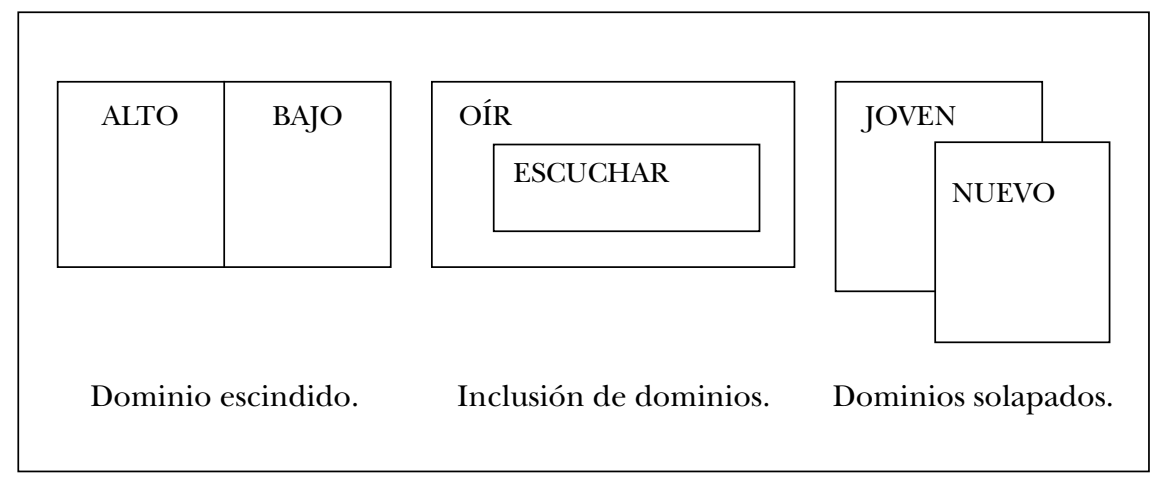

Figura 1: Esquema de los tres tipos de relaciones léxicas: «antonimia», «hiperonimia-hiponimia» $y$ «parasinonimia».

En los procesos de conceptualización están presentes una serie de habilidades cognitivas, señaladas por Croft y Cruse 2004, p. 46, manifestadas en diferentes aspectos de la experiencia, de las que dependen diversos procesos conceptuales secundarios que han sido objeto de atención por parte de la Psicología cognitiva y que encuentran aplicación también en el ámbito del procesamiento lingüístico. De ellas queremos 
destacar principalmente la «atención» (o «prominencia»), habilidad relacionada con los grados de activación de las estructuras conceptuales, en la que participan procesos como la selección o habilidad para atender a partes de nuestra experiencia relevantes para un determinado propósito o ignorar aspectos irrelevantes para tal propósito. También se vincula a esta habilidad la noción de «dominio» o «esfera de atención», en cuanto hecho accesible por un punto de referencia que funciona como foco de atención. Si trasladamos estos procesos al caso de las relaciones léxicas, observaremos que todas ellas se apoyan en distintos grados de focalización de los rasgos de similitud y contraste.

$\mathrm{Al}$ margen de la atención, también otras habilidades cognitivas ${ }^{28}$, sirven de base en la explicación del procesamiento de las relaciones léxicas. Entre ellas, se encuentra el juicio (o comparación), vinculado a procesos como la categorización y el alineamiento figura-fondo, donde el principio de contraste, que articula las relaciones léxicas, ocupa un lugar fundamental. También, la habilidad de perspectiva (o situación), no aplicable exclusivamente a los dominios espaciales sino de forma general a nuestros conocimientos, creencias y actitudes, y donde se incluyen procesos relacionados con el punto de ventaja y la orientación, se conecta con fenómenos como el de la inclusión semántica y el establecimiento de jerarquías de rasgos. La llamada habilidad de constitución, relativa a la conceptualización de la misma estructura de las entidades que componen la escena, sirve de base a la segmentación de los contenidos sémicos que posteriormente se focalizarán o se dejarán en segundo plano.

El análisis del conjunto de las relaciones léxicas a partir de la actuación del mecanismo cognitivo de focalización nos permite señalar ciertas diferencias patentes en los ámbitos de la lengua y del hablar. De este modo, observamos que, desde el punto de vista de su funcionamiento en el sistema de la lengua, mientras que en la antonimia léxica existe un proceso de focalización de los rasgos de contraste, en las otras relaciones la esfera de atención se ubica en los rasgos de similitud. Apelando a los anteriores ejemplos, en los pares alto / bajo y hombre / mujer

\footnotetext{
${ }^{28}$ Con el apoyo de la clasificación elaborada por Croft y Cruse 2004, pp. 40-73, fruto de la reformulación de propuestas previamente elaboradas tanto por parte de distintos lingüistas cognitivos como por las tradiciones filosófica y psicológica anteriores, realizamos en trabajos anteriores una propuesta articulada en torno a estas cuatro habilidades cognitivas. En ella observamos que las distintas manifestaciones de la relación antonímica tienen en común la existencia de un dominio conceptual compartido por los dos antónimos, la focalización de la atención sobre los rasgos diferenciales, con un fondo conceptual común, y la categorización de los dos miembros de la relación. Además, mientras que las habilidades de atención y juicio explican lo que podemos llamar «instinto del antónimo», las habilidades de perspectiva sirven de base a la antonimia deíctica (cerca / lejos, antes / después, abrir / cerrar) y las de constitución a la antonimia escindida (bueno / malo, frío / caliente, hombre / mujer). Cf. Varo Varo 2007, pp. 124-127.
} 
se focalizan los rasgos diferenciales «superior a la media»e «inferior a la media» $y$ «de sexo masculino»y «de sexo femenino» y en los pares oír/escuchar y joven / nuevo la atención se dirige a la esfera semántica común «percibir a través del oído»y «de poco tiempo».

No ocurre lo mismo desde el punto de vista del hablar, dado que en él la esfera de atención presenta un carácter dinámico, es decir, puede desplazarse de unos rasgos a otros a lo largo de la escena ${ }^{29}$, cambiando, así, los participantes en la comunicación el tipo de focalización. En este sentido, el establecimiento de contrastes antitéticos en el hablar entre unidades léxicas, más allá de afectar a unidades que son antónimos de lengua, también se extiende a unidades cuasisinónimas del sistema (por manifestar mayor número de semas comunes que diferenciales). Dicho fenómeno halla explicación en ciertos procesos de focalización cognitiva desarrollados por el individuo en determinados contextos o situaciones, en los que la oposición se traslada desde el nivel de los lexemas al de la articulación sémica.

En tanto que todas las relaciones léxicas se pueden focalizar desde el principio de contraste, observamos que el principio de similitud no opera con la misma eficacia en el hablar. En efecto, aunque las relaciones de hiperonimia-hiponimia pueden neutralizarse en el discurso, como consecuencia de la focalizacion de los rasgos comunes, en el caso de las relaciones de parasinonimia, a pesar de tratarse de unidades tan próximas en su significado, nunca se neutraliza su diferencia sémica en ningún contexto y en el hablar su utilización suele responder a un propósito diferenciador. Es el caso de los ejemplos puedo estar apartado, mas no ausente $^{30}$ y yo estaba conmovido, no, conmovido no, aterrado ${ }^{31}$, donde la atención se centra en la diferencia entre las unidades léxicas apartado y ausente, de un lado, y conmovido y aterrado, de otro, quedando de relieve en el contexto que la segunda unidad añade una marca más de intensidad, ausente en la primera. En cambio, de forma paradójica, los antónimos de lengua pueden llegar a cierto acercamiento semántico en determinados usos estilísticos, en los que se persigue la convivencia entre opuestos, como ocurre en el ejemplo (...) oscura luz que por tinieblas guía $(. . .)^{32}$. Estos peculiares usos, aunque escasos, nos sirven para corroborar la mayor cercanía semántica entre antónimos (pertenecientes al fin y al cabo a un mismo dominio escindido), frente al resto de relaciones (que atañen en realidad a dominios diferentes, que pueden estar solapados o incluido uno en otro). Asimismo, el hecho de que

\footnotetext{
29 Cf., en este sentido, Talmy 2000, capítulo 2.

${ }^{30}$ Quevedo, F., Poemas escogidos, Madrid, Clásicos Castalia, p. 184.

31 Bécquer, G. A., Leyendas, Barcelona, Bruguera, p. 226.

32 Cetina, G. de, Sonetos y madrigales completos, Madrid, Cátedra, p. 275.
} 
en ningún contexto puedan alternar parasinónimos (vinculados mediante una relación de equipolencia), pese a que sus rasgos comunes predominan sobre los diferenciales, pone de relieve la trascendencia del principio de contraste en la configuración del léxico.

TABLA 1: Cuadro resumen de la caracterización psicolingüística de las relaciones léxicas.

\begin{tabular}{|l|l|l|l|}
\hline $\begin{array}{l}\text { RELACIONES } \\
\text { LÉXICAS }\end{array}$ & $\begin{array}{l}\text { REPRESENTACIÓN } \\
\text { CONCEPTUAL }\end{array}$ & $\begin{array}{l}\text { PROCESOS } \\
\text { COGNITIVOS } \\
\text { (LENGUA) }\end{array}$ & $\begin{array}{l}\text { PROCESOS } \\
\text { COGNITIVOS } \\
\text { (HABLAR) }\end{array}$ \\
\hline ANTONIMIA & $\begin{array}{l}\text { DOMINIO } \\
\text { ESCINDIDO }\end{array}$ & $\begin{array}{l}\text { FOCALIZACIÓN } \\
\text { RELACIÓN } \\
\text { DE CONTRASTE }\end{array}$ & $\begin{array}{l}\text { FOCALIZACIÓN } \\
\text { RELACIÓN } \\
\text { DE CONTRASTE } \\
\text { SIMILITUD }\end{array}$ \\
\hline $\begin{array}{l}\text { HIPERONIMIA } \\
\text { HIPONIMIA }\end{array}$ & $\begin{array}{l}\text { INCLUSIÓN } \\
\text { DE DOMINIOS }\end{array}$ & $\begin{array}{l}\text { FOCALIZACIÓN } \\
\text { RELACIÓN } \\
\text { DE SIMILITUD }\end{array}$ & $\begin{array}{l}\text { FOCALIZACIÓN } \\
\text { RELACIÓN } \\
\text { DE CONTRASTE } / \\
\text { SIMILITUD }\end{array}$ \\
\hline PARASINONIMIA & $\begin{array}{l}\text { DOMINIOS } \\
\text { SOLAPADOS }\end{array}$ & $\begin{array}{l}\text { FOCALIZACIÓN } \\
\text { RELACIÓN } \\
\text { DE SIMILITUD }\end{array}$ & $\begin{array}{l}\text { FOCALIZACIÓN } \\
\text { RELACIÓN } \\
\text { DE CONTRASTE }\end{array}$ \\
\hline
\end{tabular}

\section{ASPECTOS DIFERENCIALES EN LOS PROCESOS DE COMPRENSIÓN Y PRODUCCIÓN DE LAS RELACIONES LÉXICAS}

Los datos proporcionados por la investigación del procesamiento de las relaciones léxicas muestran, además de la conexión entre cognición y lenguaje, importantes divergencias entre ellas, que nos ayudan a caracterizarlas y singularizarlas de forma más precisa.

En la investigación de los procesos conceptuales y asociativos vinculados a las relaciones léxicas, parte de las conclusiones se basan en el análisis neuronal de las respuestas manifestadas ante determinados estímulos (como los realizados por Vaughan y otros 1982, y Herrman y otros 1986), que pretenden descubrir qué elementos intervienen cognitivamente en su reconocimiento. Igualmente podemos mencionar a este respecto otros trabajos experimentales sobre el reconocimiento de sinónimos y antónimos (como el de Gazzanija y Miller 1989) o simplemente aproximaciones desde el punto de vista nocional o asociativo a 
su funcionamiento ${ }^{33}$, que coinciden en señalar notables diferencias. Incluso en el mismo marco de la antonimia, Varley 1991 establece una jerarquía en las relaciones antonímicas según el grado de dificultad en el procesamiento, desde los antónimos graduables, más fáciles de reconocer, hasta los direccionales e inversos, más complejos.

El procesamiento ha sido también analizado a través de los casos de afasia cerebral, que suelen atribuir mayor grado de complejidad a la antonimia que a la sinonimia. En esta línea, H. Gardner y otros 1978, pp. 301-317 hace ya algunas décadas desarrollaron un estudio de la comprensión y producción de antónimos en pacientes afásicos, con objeto de demostrar la existencia de una estructura interna previa al dominio significativo que se manifiesta a través de una estructura lingüística o simbólica. Al analizar la competencia antonímica o posibilidad de producir y comprender opuestos en dimensiones distintas o de establecer relaciones entre ellos, a través de diversas pruebas experimentales, descubrieron interesantes diferencias, según se tratase de antónimos canónicos o no canónicos (del tipo gato-ratón o montaña-valle), de pacientes con daños en el hemisferio derecho o en las áreas de Broca o Wernicke, de tareas de comprensión o de producción, y de verbos o sustantivos ${ }^{34}$ antónimos. Así, los afásicos de Wernicke, frente a los de Broca y a los que sufrieron daños en el hemisferio derecho, mostraron mayores dificultades en el reconocimiento de los antónimos, especialmente en el caso de los no canónicos, para los que con frecuencia proporcionaron sinónimos. Tales observaciones, sin duda, prueban la vinculación de las relaciones léxicas con áreas específicamente lingüísticas.

En diversos estudios de carácter experimental, basados en la indagación del tiempo invertido en el reconocimiento y la producción de las relaciones léxicas, se percibe una importante diferencia entre la antonimia (canónica) y el resto de relaciones. Es el caso de los trabajos realizados por Murphy y Andrew 1993, Charles y otros 1994 y Murphy 2003, pp. 136 y ss. En concreto, se descubre que los antónimos canónicos son procesados de forma más rápida que los sinónimos y los antónimos no canónicos. Entre las posibles causas explicativas de tales diferencias se aduce, como hemos visto, la coocurrencia, que facilita la asociación léxica y que afecta más a antónimos, y la relación de simetría ${ }^{35}$ entre

${ }^{33}$ Cf. Gross, Fischer y Miller 1989, Berthoneau 1993, Murphy y Andrew 1993, Charles, Reed y Derrebery 1994, y Sauborin y Libben 2000.

${ }^{34}$ Curiosamente, los afásicos de Broca estudiados por Gardner y su equipo mostraron más problemas con el reconocimiento de antónimos verbales, mientras que los de Wernicke tuvieron dificultades con el reconocimiento de antónimos sustantivos.

35 Sirva como ejemplo la caracterización de antonimia ofrecida por Andrew y Murphy 1993, pp. 304 y 305: «antonyms are words that differ on the value of one dimension such that they are equally far from some neutral point, in opposite directions, on that dimension»; "Antonyms are gradable adjectives that differ solely in one dimension, such that the values of the two adjectives on that dimension are equal distances in opposite directions from a neutral point». 
antónimos (perfectos), frente a los sinónimos y antónimos no canónicos, en los que normalmente se dan asimetrías desde el punto de vista de su combinatoria o uso contextual.

Sin embargo, la frecuente coocurrencia de unidades léxicas antónimas en el discurso, señalada como causa posible del procesamiento más rápido de dicha relación, resulta insuficiente, dado que las relaciones entre hiperónimos e hipónimos (o de inclusión semántica) son aún más frecuentes en el hablar, donde muchas veces estas unidades funcionan como sinónimos referenciales. De hecho, la sinonimia referencial en cuanto mecanismo de coherencia textual es mucho más común que la antonimia en los textos. Así pues, más que una causa, este fenómeno constituye una consecuencia de la vinculación semántica entre dichas unidades.

En cuanto a la simetría de los antónimos canónicos, frente a la asimetría presente en el resto de relaciones léxicas, hemos de advertir que también las relaciones de parasinonimia, sustentadas en la equipolencia semántica, son simétricas desde el punto de vista de los contenidos semánticos implicados. Solo en el caso de la hiperonimia-hiponimia o en el de sinónimos diasistemáticos ${ }^{36}$ (relacionados con distintas geografías, grupos sociales o estilos de lengua) se puede reconocer una asimetría vinculada a la marcabilidad semántica o al uso, respectivamente. Por otra parte, incluso en el caso de la antonimia canónica, apreciamos ciertas asimetrías como las presentes en las relaciones feo / guapo-bonito o duro / blando-tierno ${ }^{37}$.

Desde nuestra postura, el diferente tipo de procesamiento de las relaciones léxicas puede ser explicado atendiendo a las bases cognitivas que hemos propuesto más arriba. Así, en el caso de la antonimia, al tener solo que recuperarse un dominio cognitivo, el tiempo de procesamiento será menor que en los casos de la hiperonimia-hiponimia y la parasinonimia, que, pese a su vinculación, afectan a dominios distintos. Por otra parte, el procesamiento de la similitud parece requerir mayor esfuerzo cognitivo que el procesamiento del contraste, por ser este último principio mucho más efectivo. Estos datos pueden correlacionarse con los proporcionados por la investigación de la ontogénesis de las relaciones léxicas ${ }^{38}$, en los que se constata que la adquisición de la antonimia suele preceder a la de las otras relaciones, especialmente la sinonimia.

\footnotetext{
${ }^{36}$ En cualquier caso, los denominados «sinónimos diasistemáticos» no se corresponden con ninguna relación léxica, al estar sujeta su diferencia a factores no lingüísticos.

37 Estas y otras asimetrías clasemáticas detectadas en las relaciones de sinonimia y antonimia son analizadas en Varo Varo 2007, pp. 155-213.

${ }^{38}$ La ontogénesis de la antonimia es abordada en nuestro trabajo Varo Varo 2003, pp. $16-27$.
} 
TABLA 2: Resumen de los factores de adquisición y procesamiento de las relaciones léxicas estudiadas.

\begin{tabular}{|l|l|l|}
\hline RELACIONES LÉXICAS & ADQUISICIÓN & PROCESAMIENTO \\
\hline ANTONIMIA & MÁS TEMPRANA & $\begin{array}{l}\text { MENOR TIEMPO } \\
\text { DE RECONOCIMIENTO }\end{array}$ \\
\hline $\begin{array}{l}\text { HIPERONIMIA-HIPONIMIA } \\
\text { Y PARASINONIMIA }\end{array}$ & MÁS TARDÍA & $\begin{array}{l}\text { MAYOR TIEMPO } \\
\text { DE RECONOCIMIENTO } \\
\text { Y PRODUCCIÓN }\end{array}$ \\
\hline
\end{tabular}

A la luz de estas observaciones, se explica no solo la mayor facilidad en el procesamiento de las relaciones de antonimia, frente a las demás, sino también la tendencia a establecer, como hemos visto, contrastes en el hablar entre unidades próximas semánticamente. En cualquier caso, sería necesario profundizar a través del análisis de muestras reales de procesamiento léxico, en el estudio de los rasgos semántico-conceptuales evocados en las respuestas obtenidas, con objeto de conocer mejor los mecanismos cognitivos que articulan estas relaciones, así como establecer semejanzas y diferencias en cuanto al tipo de procesamiento que caracteriza a cada relación léxica.

\section{REFERENCIAS BIBLIOGRÁFICAS}

APRILE, L. (1992): «Modello di sviluppo dei fattori cognitivo-linguistici della tautologia, sinonimia e antonimia nei bambini dai 3 ai 6 anni di eta», Rassegna Italiana di Linguistica Applicata 24, 1, pp. 61-83.

BARSALOU, L. W. (1987): «The inestability of graded structure: Implications for the nature of concepts», en Neisser, U. (ed.), Concepts and conceptual development: Ecological and intellectual factors, Cambridge, Cambridge University Press, pp. 101-140.

Berthonneau, A.-M. (1993): «Avant/après. De l'espace au temps», Lexique 11, pp. 41-109.

Bolinger, D. L. (1977): Meaning and form, Londres, Longman.

BRÉAL, M. (1924): Essai de sémantique. Science des significations, $7^{\mathrm{a}}$ ed., París, Librairie Hachette, 1897.

Butters, N.; BARTOn, M. y Brody, B. A. (1970): «Role of the right parietal lobe in the mediation of cross modal associations and reversible operations in space», Cortex 6, pp. 174-190.

CARAmazzA, A. y otros (1976): «Right hemisphere damage and verbal problem solving», Brain and Language 3, pp. 41-46. 
- (1997): «Neutralización y sinonimia: la caracterización funcional de los sinónimos como elementos léxicos en oposición neutralizable», Serra Alegre, E. y otros (eds.), Panorama de la investigació lingüistica a l'Estat Espanyol. Actes del I Congrés de Lingüistica General (València, 15, 16 i 17 de febrer de 1994), vol. III: Fonètica i fonología. Semántica i pragmàtica, Valencia, Universidad de Valencia, pp. 99-106.

CASAs Gómez, M. (2002): Los niveles del significar, Documentos de Investigación Lingüística 7, Cádiz, Universidad de Cádiz.

- (2005): «Relaciones "significativas", relaciones semánticas y relaciones léxicas», Lingüistica Española Actual 27, 1, pp. 5-31.

- (2008): «El concepto de significante en el funcionalismo semántico», Romanische Forschungen 120, 3, pp. 283-306.

Chaffin, R. y Herrmann, D. J. (1984): «The similarity and diversity of semantic relations», Memory and Cognition 12, pp. 134-141.

Charles, W. G.; Reed, M. A. y Derrebery, D. (1994): «Conceptual and associative processing in antonymy and synonymy», Applied Psycholinguistics 15, 3, pp. 329-354.

ClaARK, E. V. (1972): "On the child's acquisition of antonyms in two semantic fields», Journal of Verbal Learning and Verbal Behaviour 11, pp. 750-758.

- (1987): «The principle of contrast: A constraint on language acquisition», en MacWhitney, B. (ed.), Mechanisms of language acquisition, Hillsdale, NJ, Lawrence Erlbaum Associates Inc.

- (1995): The lexicon in acquisition, Cambridge, Cambridge University Press.

Cooper, W. E. y Ross, J. R. (1975): «World order», en Grossman, R. E., San, J. L. y Vance, T. J. (eds.), Papers from the parasession on functionalism, Chicago, Chicago Linguistics Society, University of Chicago Press.

Coseriu, E. (1981): Principios de semántica estructural, $2^{a}$ ed., Madrid, Gredos.

Croft, W. y Cruse, D. A. (2004): Cognitive linguistics, Cambridge, Cambridge University Press.

Cruse, D. A. y Togia, P. (1995): «Towards a cognitive model of antonymy», Lexicology 1, 1, pp. 113-141.

CuenCA, M. J. y HiLferTy, J. (1999): Introducción a la lingüística cognitiva, Barcelona, Ariel.

Damasio, H. y otros (1996): «A neural basis for lexical retrieval», Nature 380, pp. $499-505$.

FEIDER, H. (1973): «Comparatives in early child language», Glossa 7, 1, pp. 3-20.

Fellbaum, C. (1995): «Co-ocurrence and antonymy», Internacional Journal of Lexicography 8, 4, pp. 281-303.

GARDNER, H. y otros (1978): «The appreciation of antonymic contrasts in aphasia», Brain and Language 6, 3, pp. 301-317.

Gazzaniga, M. S. y Miller, G. A. (1989): «The recognition of antonymy by a language-enriched right hemisphere», Journal of Cognitive Neuroscience 1, 2, pp. 187-193.

GRICE, H. P. (1975): «Logic and conversation», Cole, P. y Morgan, J. (eds.), Syntax and Semantics: Vol. 3: Speech acts, Nueva York, Academic Press, pp. 41-58.

Gross, D.; Fischer, U. y Miller, G. A. (1989): «The organization of adjectival meanings», Journal of Memory and Language 28, 1, pp. 92-106. 
Gross, D. y Miller, G. A. (1990): «Adjectives in WordNet», International Journal of Lexicography 3, pp. 265-277.

Hatzivassiloglou, V. (1999): Automatic acquisition of lexical semantic knowledge from large corpora: The identification of semantically related words, markedness, polarity, and antonymy, Columbia, Dissertation.

Herrmann, D. J. y otros (1986): «The role of elements of relation definition in antonym and synonym comprehension», Zeitschrift fur Psychologie mit Zeitschrift für angewandte Psychologie 194, 2, pp. 133-153.

HJelmslev, L. (1971): Prolegómenos a una teoría del lenguaje, trad. esp., Madrid, Gredos.

JACKENDOFF, R. S. (1983): Semantics and cognition, Cambridge MA, MIT Press.

Jakobson, R. (1975): Ensayos de lingüistica general, trad. esp., Barcelona, Seix Barral.

JONES, S. (2002): Antonymy. A corpus-based perspective, Londres, Routledge.

Justeson, J. S. y Katz, S. M. (1991): «Redefining antonymy: The textual structure of a semantic relation», Literary and Linguistic Computing 7, pp. 176184.

KARČEVSKIJ, S. (1929): «Du dualisme asymétrique du signe linguistique», Travaux $d u$ Cercle Linguistique de Prague 1, pp. 88-93.

Krampen, M. (1984): «Binary features and visual semantics. A study of children's drawings of different buildings types», Quaderni di Semantica 5, 1, pp. 170182.

KwON, H.-S. (1998): «A corpus-based study of English negative prefixation», 1998 TAL Proceedings, Londres, Longman.

Johnson, M. (1987): The body in the mind: The bodily basis of meaning, reason and imagination, Chicago-Londres, Chicago University Press.

Landis, G.; Herrmann, D. y Chaffin, R. (1987): «Developmental differences in the comprehension of semantic relations», Zeitschrift fur Psychologie mit Zeitschrift fur angewandte Psychologie 195, 2, pp. 129-139.

Lehrer, A. (1990): «Polisemy, conventionality, and the structure of the lexicon», Cognitive Linguistics 1, pp. 207-246.

Marcus, S. (1989), «N. S. Trubetzkoy and his followers in the study of linguistic oppositions», en Martín Vide, C. (ed.), Actas del IV Congreso de Lenguajes Naturales y Lenguajes formales (Lleida, 19-23 de septiembre de 1988), 2, Barcelona, Universidad de Barcelona, PPU, pp. 943-961.

Mettinger, A. (1994): Aspects of semantic opposition in English, Oxford, Oxford University Press.

Miller, G. A. y otros (1990): «Introduction to WordNet: An on-line database», International Journal of Lexicography 3, pp. 235-244.

- y Charles, W. G. (1991): "Contextual correlates of semantic similarity», Language and Cognitive Processes 6, pp. 1-28.

- y Fellbaum, C. (1991): «Semantic networks of English», Cognition 41, pp. 197-229.

MueHleisen, V. (1997): Antonymy and semantic range in English, Ph.D. dissertation, Northwestern University.

Muñoz NúÑEz, Mํㅡㄹ. (1999): La polisemia léxica, Cádiz, Universidad de Cádiz. 
Murphy, L. M. (2003): Semantic relations and the lexicon, Cambridge, Cambridge University Press.

- y ANDrew, J. M. (1993): "The conceptual basis of antonymy and synonymy in adjectives», Journal of Memory and Language 32, 3, pp. 301-319.

SABOURIN, L. y LibBEn, G. (2000): «Lexical processing of synonymy and antonymy: An exploration of task and word form differences», University of Alberta Papers in Experimental and Theoretical Linguistics 5, pp. 114-136.

Saussure, F. de (1980): Curso de lingüistica general, Publicado por Ch. Bally y A. Sechehaye, Barcelona, Akal.

SCHANk, R. y Abelson, R. (1977): Scripts, plans, goals and understanding, Hillsdale, Lawrence Erlbaum.

Singleton, D. (2000): Language and the lexicon: An introduction, Nueva York, Oxford, University Press.

TALMY, L. (2000): Toward a cognitive semantics, Cambridge, MA, MIT Press.

Trubetzkoy, N. S. (1973): Principios de fonología, Madrid, Paraninfo.

Ullmann, M. T. (2006): «Language and the brain», en Connor-Linton, J. y Fasold, R. W. (eds.), An Introduction to language and linguistics, Cambridge, Cambridge University Press.

VARLEY, R. (1991): «Reference, sense and antonymy in the assessment of lexical semantic abilities in aphasia», Aphasiology 5, 2, pp. 149-170.

VAro VAro, C. (2003): La polaridad en el lenguaje, Documentos de Investigación Lingüística 8, Cádiz, Universidad de Cádiz.

- (2006): «Algunas observaciones sobre la aplicación del principio de oposición en Lexicología», en Luque Durán, J. de D. (ed.), Actas del V Congreso Andaluz de Lingüistica General. Homenaje al profesor José Andrés de Molina Redondo, II, Granada, Granada Lingvistica, Serie Collectae, pp. 819-827.

- (2007): La antonimia léxica, Madrid, Arco/Libros.

VAughan, J. y otros (1982): «Cortical evoked responses to synonyms and antonyms», Memory and Cognition 10, 3, pp. 225-231.

WAugh, L. (1982): «Marked and unmarked: A choice between unequals in semiotic structure», Semiotica 38, pp. 299-318.

Wотјак, G. (1979): Investigaciones sobre la estructura del significado, Madrid, Gredos. 
